\title{
Porphysome nanoparticles for enhanced photothermal therapy in a patient-derived orthotopic pancreas xenograft cancer model: a pilot study
}

Christina M. MacLaughlin

Lili Ding

Cheng Jin

Pingjiang Cao

Iram Siddiqui

David M. Hwang

Juan Chen

Brian C. Wilson

Gang Zheng

David W. Hedley 


\title{
Porphysome nanoparticles for enhanced photothermal therapy in a patient-derived orthotopic pancreas xenograft cancer model: a pilot study
}

\author{
Christina M. MacLaughlin, ${ }^{\text {a,b,c }}$ Lili Ding, ${ }^{a}$ Cheng Jin,,${ }^{\text {a,b,d }}$ Pingjiang Cao, ${ }^{a}$ Iram Siddiqui, ${ }^{a}$ David M. Hwang, \\ Juan Chen, ${ }^{a}$ Brian C. Wilson, ${ }^{a, b, c}$ Gang Zheng, ${ }^{a, b, d, *}$ and David W. Hedley ${ }^{a, b, c, *}$ \\ aUniversity Health Network, Princess Margaret Cancer Center, 101 College Street, Toronto, Ontario M5G 1L7, Canada \\ bUniversity of Toronto, Department of Medical Biophysics, 101 College Street, Toronto, Ontario M5G 1L7, Canada \\ cPrincess Margaret Hospital, Department of Medical Oncology and Hematology, 610 University Avenue, Toronto, Ontario M5T 2M9, Canada \\ dUniversity of Toronto, Department of Pharmaceutical Sciences, 144 College Street, Toronto, Ontario M5T 2M9, Canada \\ eUniversity Health Network, Department of Pathology, 200 Elizabeth Street, Toronto, Ontario M5G 2C4, Canada
}

\begin{abstract}
Local disease control is a major challenge in pancreatic cancer treatment, because surgical resection of the primary tumor is only possible in a minority of patients and radiotherapy cannot be delivered in curative doses. Despite the promise of photothermal therapy (PTT) for focal ablation of pancreatic tumors, this approach remains underinvestigated. Using photothermal sensitizers in combination with laser light irradiation for PTT can result in more efficient conversion of light energy to heat and improved spatial confinement of thermal destruction to the tumor. Porphysomes are self-assembled nanoparticles composed mainly of pyropheophorbide-conjugated phospholipids, enabling the packing of $\sim 80,000$ porphyrin photosensitizers per particle. The high-density porphyrin loading imparts enhanced photonic properties and enables high-payload tumor delivery. A patientderived orthotopic pancreas xenograft model was used to evaluate the feasibility of porphysome-enhanced PTT for pancreatic cancer. Biodistribution and tumor accumulation were evaluated using fluorescence intensity measurements from homogenized tissues and imaging of excised organs. Tumor surface temperature was recorded using IR optical imaging during light irradiation to monitor treatment progress. Histological analyses were conducted to determine the extent of PTT thermal damage. These studies may provide insight into the influence of heat-sink effect on thermal therapy dosimetry for well-perfused pancreatic tumors. ๑ 2016 Society of Photo-Optical Instrumentation Engineers (SPIE) [DOI: 10.1117/1.JBO.21.8.084002]
\end{abstract}

Keywords: photothermal therapy; pancreatic cancer; photothermal sensitizer; porphyrin-lipid nanoparticle; orthotopic pancreas xenograft tumor model; fluorescence imaging.

Paper 160207SSPRR received Mar. 31, 2016; accepted for publication Aug. 2, 2016; published online Aug. $23,2016$.

\section{Introduction}

The development of effective treatment strategies for pancreatic cancer presents an acute healthcare challenge, largely due to complexity in the delivery of therapies that are routinely effective for other cancers. Inability to control primary tumor burden accounts for $>30 \%$ of the mortality in this disease due to frequent localization of tumors in the head of the pancreas. ${ }^{1}$ This region is immediately adjacent to the duodenum and major vasculature, notably the superior mesenteric artery, celiac trunk, and hepatic artery, and damage to such structures would result in significant treatment-associated morbidity and mortality. Surgical resection via the highly invasive pancreaticoduodenectomy (Whipple procedure) is only feasible in as few as $15 \%$ to $20 \%$ of cases. ${ }^{2}$ The delivery of radiotherapies in curative doses also has a tendency to place adjacent organs at risk of nonspecific damage. ${ }^{3}$ Chemotherapy, principally with gemcitabine or fluorouracil, is provided as a palliative treatment as it is only able to extend survival in the range of months. ${ }^{2,4}$ With a dismal 5 -year survival rate of $<6 \%$, pancreatic cancer is projected to

*Address all correspondence to: Gang Zheng, E-mail: gang.zheng@uhnres utoronto.ca; David W. Hedley, E-mail: david.hedley@uhn.ca become the fifth-leading cause of cancer-related death in North America by $2025 ;^{2}$ therefore, intensive effort must be directed toward the development of novel therapeutic approaches, specifically tailored to overcome current challenges in the treatment of this disease.

Thermal ablative therapies have shown promise in both preclinical and clinical studies as novel approaches for the focal reduction of tumor burden in cancers such as prostate, kidney, and liver. ${ }^{5}$ Local heat generation can be accomplished using several modalities, however, the most widely investigated radiofrequency ablation (RFA) and high-intensity focused ultrasound (HIFU) carry shortcomings that limit their use for ablation of tumors in the head of the pancreas. RFA can be invasive, requiring percutaneous insertion of multiple electrodes if operated in bipolar configuration, or may subject structures surrounding the tumor to hyperthermic conditions if delivered in unipolar configuration. ${ }^{3}$ Additionally, therapeutic applied voltage in RFA is highly contingent upon tissue water and ion content, and therefore, the development of treatment protocols can be complex as a result of intertumoral heterogeneity and rapid water vaporization from tissues during RFA administration. While

$1083-3668 / 2016 / \$ 25.00$ @ 2016 SPIE 
HIFU is less invasive, ultrasonic energy must still pass through organs and vessels adjacent to the pancreas, placing these structures under significant risk of damage.

Photothermal therapy (PTT) is proposed here as an alternative to other thermal ablative techniques for reduction of primary pancreatic tumor burden. PTT involves the irradiation of target tissues with near-IR (NIR) laser light causing local generation of heat due to absorbance and subsequent vibration of molecular chromophores in the treatment zone. The target temperature range for therapeutic effect is $58^{\circ} \mathrm{C}$ to $70^{\circ} \mathrm{C}$, at which threshold there is significant denaturation of proteins, and generation of coagulative necrosis within seconds. ${ }^{5-7}$ Lower temperatures require extended irradiation times for any reduction of cell viability to be observed and higher temperatures can result in rapid vaporization or carbonization of tissue that actually impedes heat diffusion through the entire tumor volume. ${ }^{6}$ Furthermore, the energy deposition rate must be optimized to account for diffusion of heat from the irradiation sight via blood circulation. 8,9 This "heat-sink effect" has been reported to significantly impact light dosimetry and the final volume of ablated tissue in PTT administration to well-vascularized tumors, such as those in the liver. ${ }^{10-12}$ While cancer cells may be inherently more susceptible to thermal damage than healthy tissues, PTT specificity can be further improved by combination with administered photothermal sensitizers. Photothermal sensitizers ideally have high NIR absorbance cross-sections as compared to endogenous tissue chromophores and principally undergo nonradiative excited state decay, thus generating heat. ${ }^{6}$ As such, high concentrations of sensitizer molecules in the tumor region can significantly decrease the laser light dose required to obtain therapeutic temperatures, as well as promote spatial confinement of heat to the tumor. ${ }^{7}$

Porphyrin-lipid nanoparticles, termed "porphysomes" are liposome-like nanoparticles composed mainly of porphyrinconjugated lipids enabling $\sim 80,000$ porphyrins to be packed in to a single $120 \mathrm{~nm}$ biodegradable particle. ${ }^{13}$ Porphysomes are used as fluorescence imaging contrast agents and photothermal sensitizers in the present investigation of pancreatic cancer PTT because they bring the following distinct advantages over small molecule porphyrin agents: (1) high-density incorporation of porphyrin molecules imparts porphysomes with enhanced photonic properties for imaging and phototherapy; (2) the enhanced permeability and retention (EPR) effect may be exploited for optimal accumulation of porphysomes in the tumor region, thus accomplishing specific, high-payload porphyrin delivery. While porphyrin-based photosensitizers have been used in early stage clinical trial for photodynamic therapy (PDT) of pancreas tumors, ${ }^{14}$ our approach enables the use of temperature-based image guidance to minimize nonspecific damage. Additionally, PTT may be more universally applied than PDT to pancreatic tumors, which are often observed to have hypoxic regions. ${ }^{15,16}$

Nanoparticles of many different materials have been engineered for PTT enhancement to take advantage of EPR-mediated tumor delivery and large optical absorbance crosssections. Inorganic particles including rare earth-doped upconversion nanocrystals ${ }^{17}$ and carbon nanotubes, ${ }^{18}$ as well as organic nanoparticles, such as polymer nanocarriers, ${ }^{19}$ have demonstrated efficacy as photothermal transducers. Plasmonic nanoparticles in particular are attractive for PTT $^{20}$ and have been widely investigated in a number of tumor models. The surface plasmon resonant wavelength is facilely tunable through choice of nanoparticle composition, size, and shape. ${ }^{21,22}$ Gold is frequently used for engineering resonances in the NIR to minimize tissue absorbance of excitation light, thereby increasing the attainable treatment depth penetration. Moreover, the ratio of absorbance to scattering in their optical extinction can be easily optimized so that plasmonic nanoparticles are efficient photothermal transducers. These alternative nanoparticle materials carry disadvantages including difficulty in elimination following treatment because species with diameters $>\sim 5 \mathrm{~nm}$ cannot be removed via renal excretion. ${ }^{23}$ Moreover, nanoparticles synthesized from exogenous materials introduce the possibility of toxicity and long biodegradation times. Porphysomes overcome the aforementioned limitations as they are composed principally of biological materials while still interacting very strongly with light. Their optical absorbance cross-section of $10^{8} \mathrm{M}^{-1} \mathrm{~cm}^{-1}$ at $680 \mathrm{~nm},{ }^{13}$ approaches cross-sections observed for plasmonic nanoparticles. ${ }^{24}$

The feasibility of using porphysome-enhanced PTT for reduction of primary pancreatic tumor burden is investigated here using a highly clinically relevant cancer model Ontario Cancer Institute Pancreas 23 (OCIP23). This model, established via implantation of a patient-derived tumor xenograft at the orthotopic site, has been shown to closely recapitulate the morphology, genetic characteristics, and density of supportive stroma that are observed in the original patient tumor. ${ }^{15,16,25}$ Therefore, investigation of PTT using OCIP23 presents a unique opportunity for preclinical evaluation of the feasibility of multiple aspects of porphysome-mediated PTT. The ability of nanoscale particles to permeate dense stroma surrounding pancreatic tumors for accumulation therein has been reported elsewhere as a challenge in the delivery of various therapeutics to treat this disease. ${ }^{26}$ Effective light delivery and generation of therapeutic temperatures with reproducible light dosimetry must also be demonstrated for pancreatic tumors. Furthermore, OCIP23 also enables the study of whether the heat-sink effect may play a role in light dosimetry for tumors of this well-perfused organ. While PTT has been studied using ex vivo pancreas tissue $^{27,28}$ and photosensitizer-enhanced PTT investigated in both pancreatic cancer cell lines ${ }^{29-31}$ and subcutaneous cellline xenograft mouse models, ${ }^{32}$ this proof-of-concept study is the first in vivo demonstration of PTT for orthotopic pancreas tumor ablation.

\section{Materials and Methods}

\subsection{Porphyrin-Lipid Nanoparticles}

Porphysomes were prepared as previously described. ${ }^{8}$ Briefly, a desiccated lipid film was formed by passing a gentle stream of nitrogen gas over a suspension of lipids in chloroform [55:40:5 molar ratio of pyropheophorbide lipid (synthesized in-house): cholesterol (Avanti Polar Lipids, Alabaster, Alabama): distearoyl-glycero-3-phosphoethanolamine-N-methoxy(polyethylene glycol) (2 kDa PEG-PE, Avanti Polar Lipids)]. Residual solvent was removed from the film under vacuum for $1 \mathrm{~h}$ prior to rehydration with phosphate buffered saline (PBS) (150-mM $\mathrm{NaCl}, 10-\mathrm{mM}$ phosphate, $\mathrm{pH}$ 7.4) for production of porphysome nanoparticles. The lipid suspension was subjected to five freezethaw cycles and particles formed using high-pressure extrusion (LIPEX Thermobarrel Extruder, Northern Lipids Inc, Burnaby, British Columbia, Canada) by 10 passes through double polycarbonate membranes with 100 -nm pore size at $70^{\circ} \mathrm{C}$. Porphysome dose for fluorescence imaging and PTT was determined using 
optical absorbance at $410 \mathrm{~nm}$ (CaryWin UV/Vis, Agilent Technologies, Mississauga, Ontario, Canada) of the porphysome lipid suspension in methanol and a previously determined molar extinction coefficient for the porphyrin-lipid $\left(97,000 \mathrm{M}^{-1} \mathrm{~cm}^{-1}\right.$ at $410 \mathrm{~nm})$. Porphysomes were prepared and stored under sterile conditions until use.

\subsection{Animal Studies}

All animal studies were carried out using institutionally approved protocols (Ontario Cancer Institute, University Health Network, Toronto, Ontario, Canada).

\subsection{Establishment of the Orthotopic Pancreas Tumor Xenograft Model}

Models were established as described elsewhere. ${ }^{15}$ Briefly, primary human pancreas tumor tissue for xenograft was obtained from the University Health Network Tumor Tissue Bank according to institutional human ethical guidelines. Tissue bank stocks were established by implanting fresh pancreatic tumor samples that were superfluous to diagnostic needs as subcutaneous xenografts in the flanks of three severe combined immunodeficiency (SCID) mice within $2 \mathrm{~h}$ of surgical excision. An early passage number of the model was cryopreserved and orthotopic xenograft tumor models for the present study generated using tissue from an early passage donor mouse. Typically, a single donor mouse provides sufficient tissue to generate 20 pancreas tumor xenograft mouse models. A number of OCIP models have been established using primary tissues with varying morphology, however, OCIP23 was specifically chosen for photosensitizer-mediated PTT experiments for its high growth rate and moderate stromal density as compared to other orthotopic OCIP tumor models.

For orthotopic xenograft models for porphysome-mediated fluorescence imaging and PTT experiments, adult SCID mice, (5 weeks old, $25 \mathrm{~g}$ average weight, obtained from Charles River) were placed under general anesthesia using $2 \%$ isoflurane in oxygen. A small incision was made in the upper left abdomen, the pancreas exposed, and a small tumor tissue fragment stitched on to the head of the pancreas. Tumor size was monitored by palpation for the first 5 weeks following implantation, with more precise diameter measurements using magnetic resonance imaging, thereafter. Two-dimensional (2-D) MR images of the mouse abdomen in the axial plane were collected using the respiratory-gated T2-weighted Rapid Acquisition Relaxation Enhancement (RARE) technique. RARE is a multiple spinecho technique that creates an image at a single-effective echo time. As the signals from each of the spin-echoes are used to generate the single image, the image acquisition time is accelerated. The acceleration factor is termed the RARE factor. In this protocol, the echo time was $8 \mathrm{~ms}$, the effective echo time was $40 \mathrm{~ms}$, and the RARE factor was 10 .

Tumor models were selected for systemic porphysome injection, fluorescence imaging, and administration of PTT when the tumor had reached $0.5-\mathrm{cm}$ diameter in one dimension as determined by manual measurements of axial T2 MR images of the mouse abdomen [Fig. 1(a)], which occurred $\sim 6$ weeks postimplantation. This tumor size was deemed optimal for use because it is sufficiently large for delivery of laser irradiation using a free-space beam while larger tumors were observed to contain significant volumes of endogenous necrosis, which may impact (a)

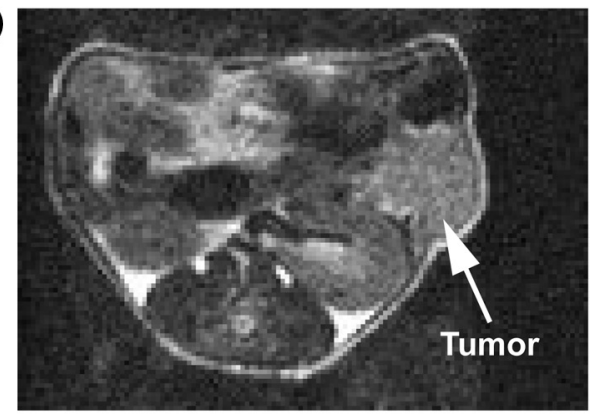

(b)

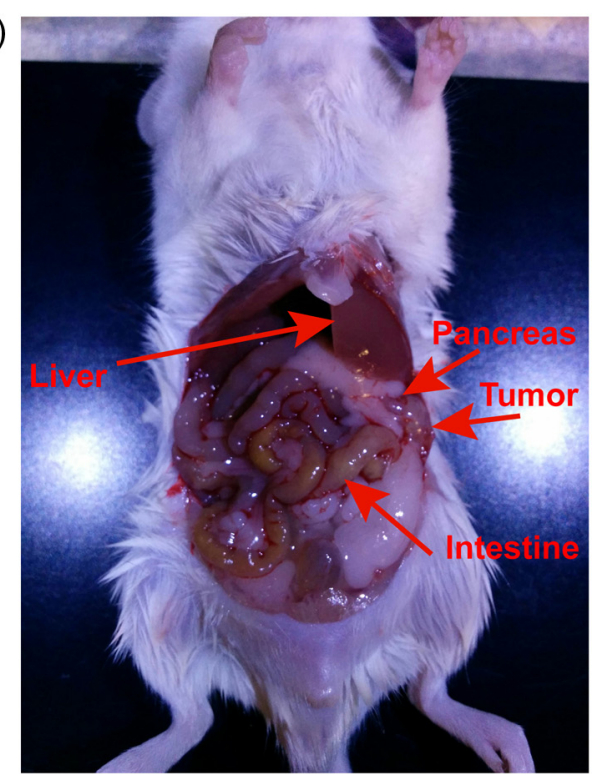

Fig. 1 Location of the orthotopic xenograft tumor in OCIP23 model. (a) T2 RARE sequence image in the axial plane of an OCIP23 mouse six weeks following tumor implantation $(32 \times 20 \mathrm{~mm}$ field-of-view; $160 \times 100$ matrix size). (b) Brightfield image of OCIP23 mouse with abdominal organs exposed to visualize location of pancreas and orthotopic tumor.

heat diffusion, as well as convolute tissue microstructure analysis.

\subsection{Ex Vivo Fluorescence Imaging}

To evaluate whether porphysomes accumulated in orthotopic xenograft OCIP23 tumors, mice were subjected to systemic tail vein injection of $10 \mathrm{mg} \cdot \mathrm{kg}^{-1}$ porphysomes suspended in $150 \mu \mathrm{L}$ PBS, under general anesthesia using $2 \%$ isoflurane in oxygen. About $24 \mathrm{~h}$ following porphysome administration, mice were sacrificed, organs of interest excised and placed in 24-well plates for imaging immediately afterward. Fluorescence imaging was conducted using excised tissues because both the deep-seated nature and placement of the tumor relative to intestines precluded transdermal and in situ tumor imaging, respectively. Ex vivo tissue fluorescence image cubes were collected using 616- to 661-nm bandpass excitation and 675-nm longpass emission optical interference filters. A liquid crystal tunable filter scanned a range of 670 to $900 \mathrm{~nm}$ and images were collected in 10-nm increments each with 200-ms integration time. Each pixel in the final 2-D image resultantly contained a fluorescence spectrum emitted from that spatial location in the sample (Maestro, Caliper Sciences, Waltham, Massachusetts) $(n=7$ positive, $n=5$ control). Fluorescence from porphysomes was 
isolated from tissue background using least-squares spectral fitting (Maestro 2.2 software, Cambridge Research and Instrumentation Inc., Woburn, Massachusetts) with tissue autofluorescence, animal diet autofluorescence, and porphyrin-lipid spectra used as input components.

\subsection{Biodistribution}

Following ex vivo fluorescence imaging, excised tissues were assayed $(n=4)$ for semiquantitative determination of percent injected porphysome dose per gram of tissue in the orthotopic tumor, pancreas, kidney, spleen, heart, lungs, liver, kidneys, muscle, adrenal glands, brain, and intestine. Tissues were suspended in PBS to a concentration of $\sim 30 \mathrm{mg} \cdot \mathrm{mL}^{-1}$ and manually homogenized on ice. The adrenal glands were suspended in PBS at lower concentrations due to the small mass of adrenal tissue available in SCID mice. Triton X-100 was added to the homogenate to a final concentration of $1 \% \mathrm{v} / \mathrm{v}$. Each sample was vortexed for $2 \mathrm{~min}$ to extract porphyrin-lipid from tissues and dissociate the nanoparticle structure. Nanoparticle dissociation is required to calculate porphysome tissue accumulation based on fluorescence intensity, because densely packed porphyrin molecules are contact quenched in the intact porphysomes. ${ }^{13}$ Homogenized suspensions were centrifuged at 13,200 rpm for $15 \mathrm{~min}$ to remove tissue debris and extracted homogenate aliquoted into 96 well plates for fluorescence measurements. Samples were excited at $410 / 10 \mathrm{~nm}$ to correspond with the pyropheophorbide-lipid Soret band absorbance and fluorescence intensity at $670 \mathrm{~nm}$ used to calculate percent injected dose, per gram (\%ID $\left.\cdot \mathrm{g}^{-1}\right)$ for each tissue. Student's $t$-test was performed (Igor Pro 6.0.3, Wavemetrics, Portland, Oregon) on fluorescence measurements from the pancreas and pancreatic tumor $(n=4)$ and differences were considered statistically significant at $p<0.05$.

\subsection{Photothermal Therapy Treatments}

The same porphysome dose used for fluorescence imaging and biodistribution was also employed for the study of photothermal sensitizer-enhanced PTT $\left(10 \mathrm{mg} \cdot \mathrm{kg}^{-1}, 24 \mathrm{~h}\right.$ after tail vein injection). Laser irradiation was conducted under general anesthesia with $2 \%$ isoflurane in oxygen for porphysome-injected $(n=3)$ and control-uninjected $(n=2)$ mice. To expose the pancreas for laser irradiation, an incision was made along the midline of the abdomen $(10 \mathrm{~mm})$ and continued with a left-side transverse incision $(7 \mathrm{~mm})$.

For laser treatment, the emission from a free-space 671-nm laser (diode-pumped solid state, LaserGlow Technologies, Toronto, Ontario, Canada) was adjusted to $750-\mathrm{mW}$ power using a variable neutral density filter (Thor Labs, Newton, New Jersey) and $\sim 0.7-\mathrm{cm}$ beam diameter spot size, for a total power density of $1.95 \mathrm{~W} / \mathrm{cm}^{2}$ at the treatment plane. Total fluence to be used for evaluating therapeutic response between treatment groups was established as the irradiation time required to increase the pancreatic tumor surface temperature to $70^{\circ} \mathrm{C}$ in porphysome-injected mice. The tumor surface temperature during irradiation was monitored using a calibrated optical IR camera with real-time temperature measurement function on its display (Mikroshot, LumaSense Technologies, Santa Clara, California). Strips of anodized aluminum were placed over tissues adjacent to the pancreas that were exposed by the abdominal incision to attenuate light scattering with the goal of preventing nonspecific tissue damage. In the case of samples where the heat-sink effect was reduced, the tumor support was also protected from the incident laser beam using anodized aluminum strips. Prior to use, it was established that significant heat generation did not occur in the anodized Al during exposure to laser fluences relevant to the present study. Plots of temperature increase over time were prepared using the maximum temperature from an region of interest placed over the tumor location in a series of IR images of mice collected at 5- to 10-s intervals during laser treatment (MikroSpec 4.0 software, LumaSense Technologies).

For proof-of-concept investigation of the impact of heat-sink effect on light dosimetry, the tumors of two mice (one porphysome injected, one uninjected control) were also raised slightly from their natural seat in the abdomen through careful placement of a cotton swab as a support underneath the tumor. Strips of anodized aluminum were placed over tissues adjacent to the pancreas that were exposed by the abdominal incision to attenuate light scattering with the goal of preventing nonspecific tissue damage. In the case of samples where the heat-sink effect was reduced, the tumor support was also protected from the incident laser beam using anodized aluminum strips.

\subsection{Histological Analyses}

Tumors were excised $1 \mathrm{~h}$ following laser irradiation and immediately fixed in 10\% PBS-buffered formalin. The fixed tissues were placed in cassettes such that serial sections were oriented orthogonally to the plane of laser irradiation. Prior to analysis, tissues were paraffin-embedded, sliced, and mounted on microscopy slides. Serial sections were stained with hematoxylin and eosin for morphological analysis.

Tissues were examined by a single pathologist, blind to sample treatment for the presence of thermal damage. Thermal damage was defined on $\mathrm{H} \& \mathrm{E}$ stained sections as distorted tissue with cells having eosinophilic appearing nucleus and cytoplasm, irregular nuclear boundaries, and loss of resolvable chromatin structure. If the presence of damage was confined solely to the edges of the tissue slice, the irregular morphology was attributed to mechanical damage incurred during sample handling and slide preparation. Brightfield images were collected using an Olympus VS120 semiautomated slide scanning microscope with Sony XM-10 camera (Olympus Canada Inc., Richmond Hill, Ontario, Canada).

For fluorescence imaging, tumors from both mice that were injected with porphysomes, as well as uninjected control mice, were immediately immersed in optimal cutting temperature gel in plastic tissue cassettes after removal and subsequently snap frozen in liquid nitrogen. Only tumors that had not received light treatment were used for fluorescence imaging because laser irradiation is expected to photobleach the porphyrin moieties, which would be used for imaging the spatial distribution of porphysome accumulation. Frozen blocks were sliced and treated with 4',6-diamidino-2-phenylindole (DAPI) nuclear stain to allow correlation of the distribution of porphysomes within the tumor. Images were collected using the same semiautomated slide scanning microscope. For imaging DAPI fluorescence, 387/15-nm bandpass excitation and 440-/45-nm bandpass emission filters were used. A 649-/20-nm bandpass excitation and 685-/33-nm emission filters were used for imaging fluorescence from porphyrin lipid. Images were pseudocolored and intensity scaled using CellSens software (Olympus Canada Inc.). 


\section{Results and Discussion}

\subsection{Development of Ontario Cancer Institute Pancreas 23 Cancer Model}

Representative axial T2 MR and brightfield images in Fig. 1 show the placement of orthotopic xenografts in the mouse abdomen. Once OCIP23 tumors had grown to be sufficiently large for PTT, they were seated adjacent to the spleen, liver, and intestines. Some heterogeneity among mice was observed in the extent to which the tumor had invaded these tissues as determined macroscopically during organ excision posttreatment and sacrifice, as well as in the histological analyses of these surrounding organs (not shown). Additionally, there was variability observed in the relative position of the tumor and the pancreas to the anterior of the mouse along the sagittal plane, as well as how closely seated the tumor was along this same plane to the skin. This heterogeneity among mice increased the complexity of treatment development, however, also served to strengthen the clinical relevance of this study.

\subsection{Accumulation of Porphysomes in Ontario Cancer Institute Pancreas 23 Orthotopic Pancreas Xenograft Tumors}

Prior to development of PTT protocols, macroscale fluorescence imaging of excised organs, microscale fluorescence imaging of tumor tissue slices, and fluorescence intensity measurements from homogenized tissues were used to determine whether porphysomes accumulated in OCIP23 orthotopic pancreas tumors at the 24 -h time point after systemic injection. About $24 \mathrm{~h}$ postinjection was chosen as the imaging and treatment time point in this study because it has shown to be effective for porphysome tumor accumulation and PTT sensitization in other mouse models of cancer, ${ }^{33}$ though optimization for this particular model would be an important variable for further study.

Ex vivo fluorescence images of excised organs from porphysome-injected and untreated control mice, respectively, were compared to visualize whether porphysomes accumulated in pancreas tumors, as well as various organs of interest (Fig. 2). Porphyrin-lipid fluorescence was observed on the exterior surfaces of the tumor and, to a lesser extent, the spleen and liver of injected mice [Fig. 2(a)]. These observations are consistent with previous studies of porphysome biodistribution in other small animal models ${ }^{13,34}$ and expected for nanoparticles that are too large to be eliminated via renal excretion. Autofluorescence background subtraction and intensity thresholding of ex vivo fluorescence images from control mice did not show any intensity consistent with the porphyrin-lipid spectrum. Therefore, any signal above baseline in ex vivo images of porphysome-injected mice could be attributed to the presence of porphyrin-lipid in each organ.

Distribution of porphysomes in the tumor microstructure was examined by fluorescence microscopy of tumor tissue slices following ex vivo imaging [Fig. 2(b)]. DAPI staining was conducted to visualize nuclei and thereby evaluate the colocalization of porphyrin-lipid fluorescence with tumor tissue. Porphyrin-lipid fluorescence was observed in the OCIP23 tumor microstructure. Confirmation that porphysomes accumulated therein is notable for the ability of nanoscale particles to pass through stroma and access the tumor in this clinically relevant model.
Semiquantitative measurements of porphysome biodistribution as percent injected dose per gram from tissues of interest in the OCIP23 model were collected by measuring porphyrin-lipid fluorescence intensity at $670 \mathrm{~nm}$ from tissue homogenate $(n=4)$ (Fig. 3). An average of $6.6 \% \pm 2.8 \% \mathrm{ID} \cdot \mathrm{g}^{-1}$ was recorded from the orthotopic tumor though there is a high degree of variability in this measurement among mice. As previously mentioned, the orthotopic xenograft tumors are somewhat variable in their development relative to other abdominal structures and may correlate to differences in tumor vascularization among mice. Despite the variability in porphysome accumulation between tumors of different mice, the tumor displayed a statistically significant greater $\% \mathrm{ID} \cdot \mathrm{g}^{-1}$ than surrounding pancreas $(* p<0.05)$. Porphysome specificity for the tumor is of particular concern in the development of focal treatments for reduction of pancreatic tumor burden due to close proximity to vital organs and extensive involvement of vasculature. The lower standard deviation for averaged accumulation in all tissues of interest with the exception of the tumor further supports the hypothesis that variable tumor growth results in differences in porphysome accumulation therein. As observed by ex vivo tissue imaging, porphysomes appreciably accumulate in the spleen and liver at $6.0 \pm 1.8$ and $2.9 \pm 0.4 \mathrm{ID} \cdot \mathrm{g}^{-1}$, respectively. Unlike ex vivo imaging, a significant measurement of $4.5 \pm 0.8 \mathrm{ID} \cdot \mathrm{g}^{-1}$ is recorded from the adrenal glands, however, this bears similarity to porphysome biodistribution in other small animal models. 8,22

Three different fluorescence measurement methods indicated that porphysomes accumulate in the tumor of OCIP23 orthotopic models, therefore, any enhancement of heat generation during PTT in nanoparticle-injected mice with respect to heat generation in control uninjected mice may be attributed to the presence of porphyrin photosensitizers therein.

\subsection{Laser Irradiation of Orthotopic Pancreatic Xenograft Tumors}

Upon confirmation that porphysomes reproducibly accumulated in orthotopic pancreas tumors, the identification of optimal light dosimetry for selective thermal ablation of porphysome-treated tumors was undertaken. Identification of optimal incident laser power for irradiation is a compromise between sufficiently slowenergy deposition such that selectivity in the rate of temperature increase between porphysome-treated and untreated tumors is achieved, while simultaneously balancing the requirement that energy deposition is rapid enough to achieve therapeutic temperatures despite heat diffusion through tissue and vasculature away from the site of administration. This is of particular relevance in the case of well-vascularized tumors where significant diffusion of thermal energy from the treatment site could impact the identification of light doses with reproducible results. While tumors were irradiated using $1 \mathrm{~W}$ and $500 \mathrm{~mW}$ incident laser power in preliminary trials, it was observed that these laser powers heated the porphysome-treated and -untreated tumors at the same rate, or required excessively long irradiation times to reach target temperature, respectively (data not shown).

The laser power parameters chosen for this study were observed to heat OCIP23 orthotopic tumors within clinically relevant irradiation times ( $\sim 2 \mathrm{~min})$, and some selectivity occurred between the rate of temperature increase in the presence and absence of photothermal sensitizers with matched laser dosimetry (Fig. 4). As only the temperature at the tumor surface was measureable using this detection method, the treatment target 


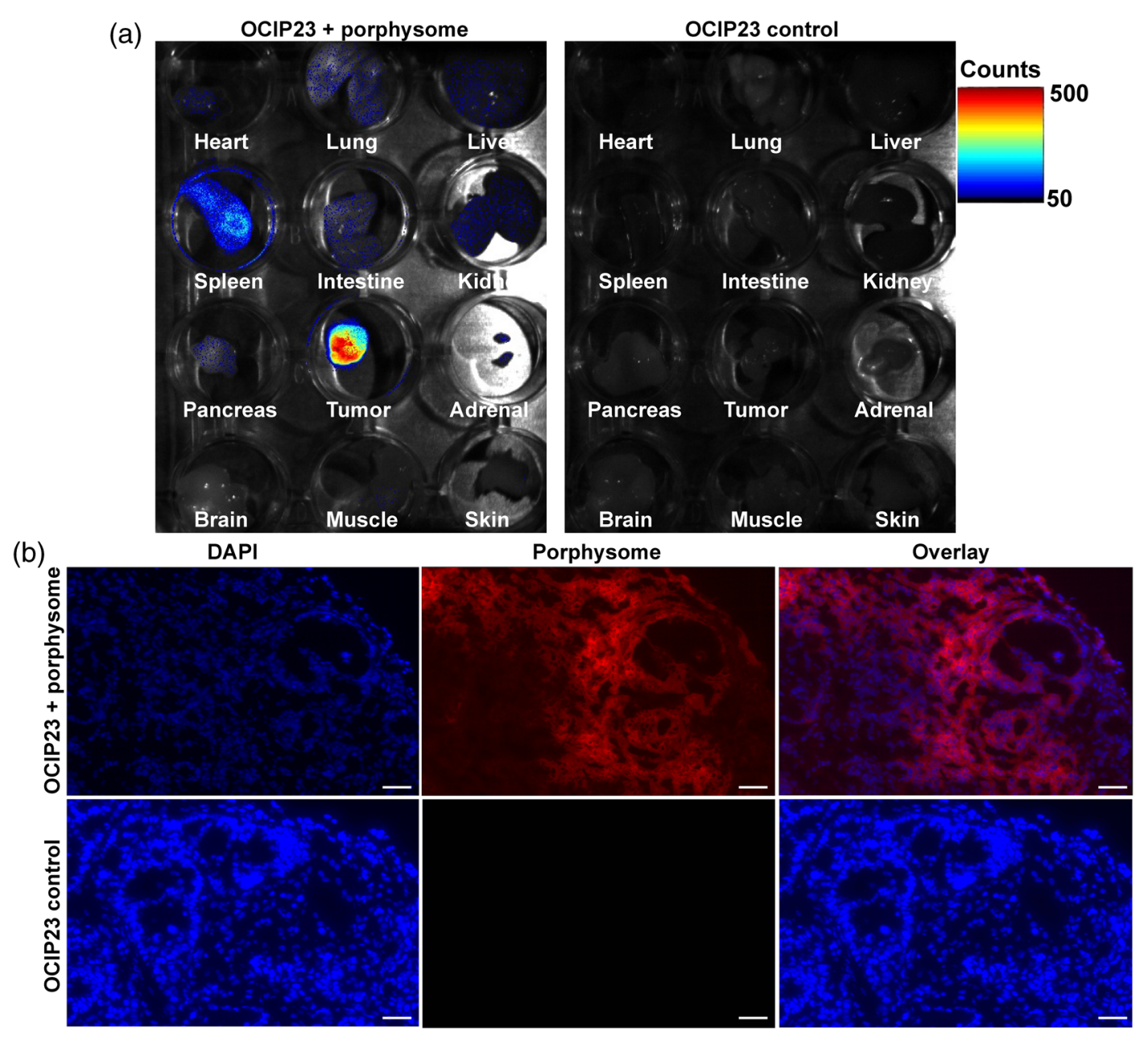

Fig. 2 Visualization of porphysome tumor accumulation in OCIP23 using fluorescence microscopy. (a) Representative ex vivo images of porphyrin-lipid fluorescence from freshly excised organs of OCIP23 mice $24 \mathrm{~h}$ following $10 \mathrm{mg} \cdot \mathrm{kg}^{-1}$ porphysome tail vein injection and no porphysome injection control as indicated. Fluorescence intensity scale shown in upper right. Fluorescence images are overlaid with monochrome bright field images of various tissues in 24-well plates. (b) Representative 60x images of pancreas tumor tissue slices from different treatment groups as labeled. DAPI pseudocolored in blue (excitation filter: $387 / 15 \mathrm{~nm}$, emission filter: $440 / 45 \mathrm{~nm}$ ), porphyrin-lipid pseudocolored in red (excitation filter: 649/40 nm, emission filter: $685 / 33 \mathrm{~nm}$ ). Scale bar $50 \mu \mathrm{m}$.

was chosen to be $70^{\circ} \mathrm{C}$ under the assumption that heat diffusion through underlying tumor tissue would result in some volume reaching $>58^{\circ} \mathrm{C}$, which is required for irreversible protein denaturation and coagulative necrosis. While $70^{\circ} \mathrm{C}$ is above the

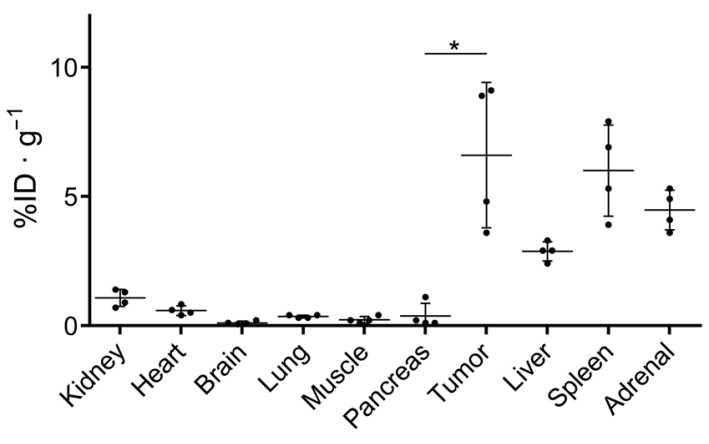

Fig. 3 Porphysome biodistribution as determined by normalizing porphyrin-lipid fluorescence intensity to mass of homogenized tissues of interest for mice sacrificed $24 \mathrm{~h}$ post tail vein injection of $10 \mathrm{mg} \cdot \mathrm{kg}^{-1}$ porphysomes $(n=4)$. Excitation $410 / 10 \mathrm{~nm}$; emission intensity measured at $670 \mathrm{~nm}\left({ }^{*} p<0.05\right)$. therapeutic target at which necrosis is expected to occur within seconds, it is still significantly below temperatures at which tissue vaporization and carbonization occur. ${ }^{6}$ Tumors of mice injected with porphysomes reached higher temperatures than the tumors of control mice subjected to identical light dose [Fig. 4(b)], though some similarity existed in the rate of temperature increase between porphysome-injected and laser control mice. As the laser irradiation times are relatively short, to induce thermal tissue damage the irradiated tissue must reach $\sim 62^{\circ} \mathrm{C}$ or higher temperatures, at which point coagulative necrosis is nearly instantaneously induced. ${ }^{5-7}$ The tumor surface temperatures attained for porphysome injected mice by a laser fluence of $468 \mathrm{~J} / \mathrm{cm}^{2}$ ranged $\sim 69^{\circ} \mathrm{C}$ to $73^{\circ} \mathrm{C}$, which is more than sufficient to generate instant tissue thermal damage. The data from PTT of two control mice plotted in Fig. 4 (b) reached $\sim 61^{\circ} \mathrm{C}$ and $\sim 45^{\circ} \mathrm{C}$ at the treatment surface, respectively, which is close to, yet still below the threshold for coagulative necrosis. While hyperthermia has been studied for destruction of tumor tissue, therapeutic effect is observed upon maintenance at hyperthermic temperatures for longer exposure times than the 220-s laser irradiation used here. ${ }^{35,36}$ Some variability in the rate of temperature 

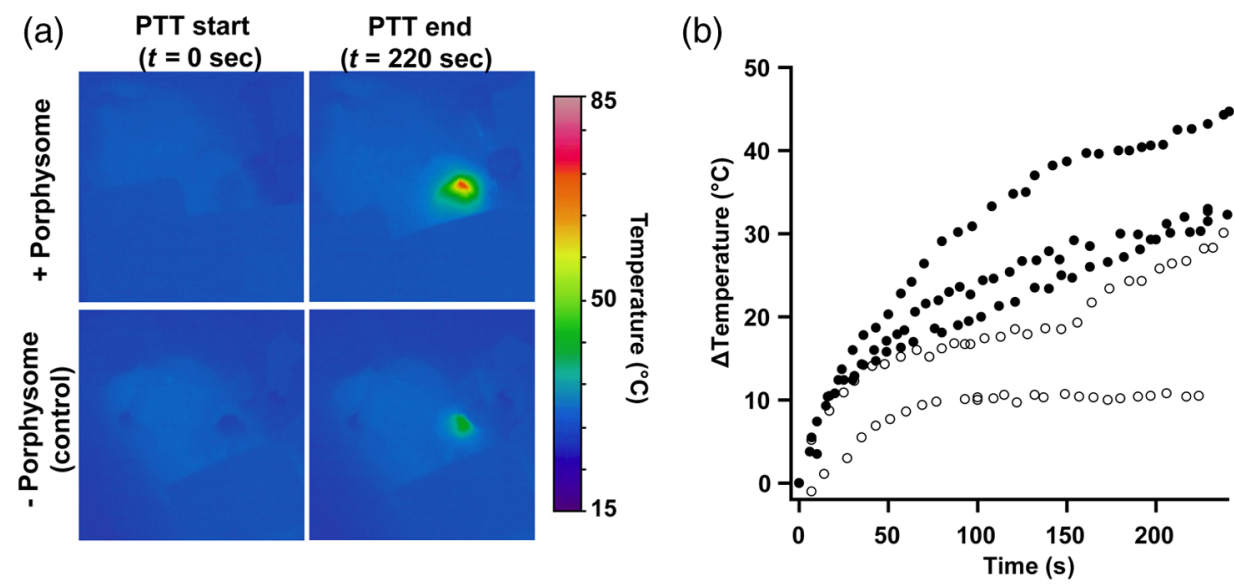

Fig. 4 Tumor region temperature increases during laser irradiation. (a) Representative heat maps collected using IR thermal camera at start and end of laser irradiation $\left(468 \mathrm{~J} / \mathrm{cm}^{2}\right.$ over $\left.220 \mathrm{~s}\right)$ for porphysome-injected and control mice, as labeled. (b) Tumor surface temperature measurements at 5- to 10-s intervals during laser irradiation for porphysome-injected (filled circle) $(n=3)$ and control (unfilled circle) $(n=2)$ mice. Each curve represents tumor surface temperatures from a single OCIP23 mouse over the course of irradiation.

increases among mice within each treatment group was observed, however, this may be attributed to differences in both the exact position and degree of invasiveness between each tumor. Refinement of the PTT administration protocol to optimize energy deposition rate, as well as to deliver light directly to the tumor via needle-guided percutaneous optical fiber is expected to enable increased discrimination between treatment groups in terms of the temperature increases observed with matched light dosimetry, thereby improving the safety and efficacy of this technique.

A proof-of-concept investigation into the impact of heat-sink effect on light dosimetry for pancreatic cancer PTT was undertaken by administering laser irradiation to tumors that were raised slightly from their natural seat in the abdomen through careful placement of a support underneath the tumor. When tumors subjected to laser irradiation were raised, the tumor tissue surface temperature reached the treatment endpoint target in a shorter time for each of one porphysome-injected and one uninjected control mice. A total light dose of $137 \mathrm{~J} / \mathrm{cm}^{2}$ was required to raise tumor surface temperature to $70^{\circ} \mathrm{C}$ for one porphysome-injected mouse when the tumor was raised within the abdomen as compared to the higher $468 \mathrm{~J} / \mathrm{cm}^{2}$ dose necessary to reach therapeutic target for tumors exposed via incision only. Notably, selectivity in temperature increase between the porphysome injected and the control mouse was still observed in the former treatment condition (Fig. 5). Furthermore, the $137-\mathrm{J} / \mathrm{cm}^{2}$ light dose required to reach the $70^{\circ} \mathrm{C}$ target treatment temperature is more consistent with light doses previously reported for efficacious porphysome-enhanced PTT in subcutaneous tumor mouse models $\left(\sim 100 \mathrm{~J} / \mathrm{cm}^{2}\right)$ that are expected to be less well perfused than the orthotopic pancreas tumors. ${ }^{21}$

In addition to monitoring tumor surface temperature, PTT efficacy was evaluated by histological analyses. H\&E stained tumor slices from each treatment group were reviewed for the presence of thermal damage in tumor morphology (Fig. 6). OCIP23 pancreas adenocarcinoma appears as aberrantly formed duct cells, often with necrotic debris in the center of the ducts. Healthy mouse acinar cells appear darker on H\&E stained tissue slices and are interspersed within the xenograft tumor cells. Tissue structural distortion, loss of nuclear structure, and eosinophilic cytoplasm were observed in tumors of mice treated with both porphysomes and laser irradiation, while such changes in tissue morphology were absent from tumors of mice subjected to laser irradiation only. The observed thermally induced morphological changes were found to be similar to those reported elsewhere for ablative therapies such as RFA and tissue resection procedures involving electrocautery. ${ }^{37,38}$

Due to the invasiveness of pancreas PTT as administered under the current study design, induction of apoptosis or longterm expression of stress and proliferation markers could not be assayed. Unfortunately, this precluded the identification of the thermal treatment marginal zone, where local temperatures are raised but the therapeutic ablative threshold is not reached.

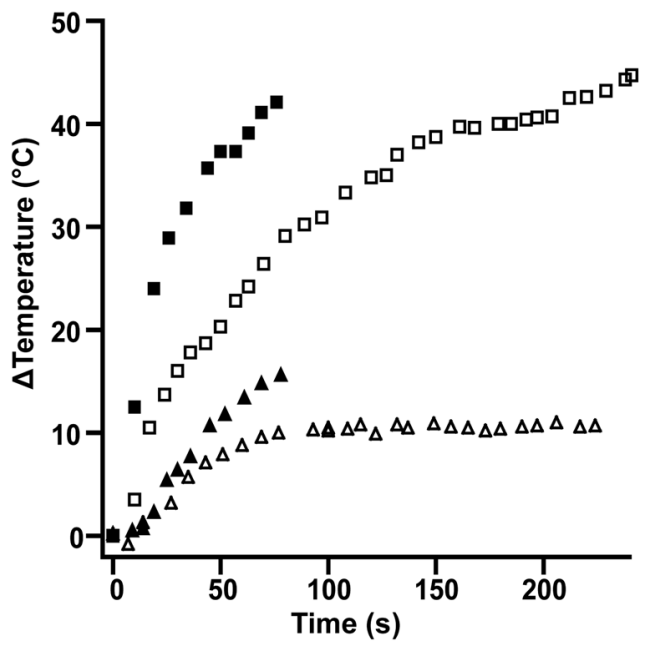

Fig. 5 Influence of the heat-sink effect on laser dosimetry via comparison of rate of tumor surface temperature increase during laser irradiation between tumors raised from natural position $\left(137 \mathrm{~J} / \mathrm{cm}^{2}\right.$ over $70 \mathrm{~s}$ to reach $70^{\circ} \mathrm{C}$ for porphysome-injected mouse) and tumors remaining in situ $\left(468 \mathrm{~J} / \mathrm{cm}^{2}\right.$ over $220 \mathrm{~s}$ to reach $70^{\circ} \mathrm{C}$ for porphysome-injected mice). Each curve represents tumor surface temperatures from a single OCIP23 mouse over the course of irradiation [filled square: porphysome-injected, raised tumor $(n=1)$; unfilled square: control, raised tumor $(n=1)$; unfilled triangle: porphysome-injected, in situ tumor $(n=1)$; unfilled triangle: control, in situ tumor $(n=1)]$. 


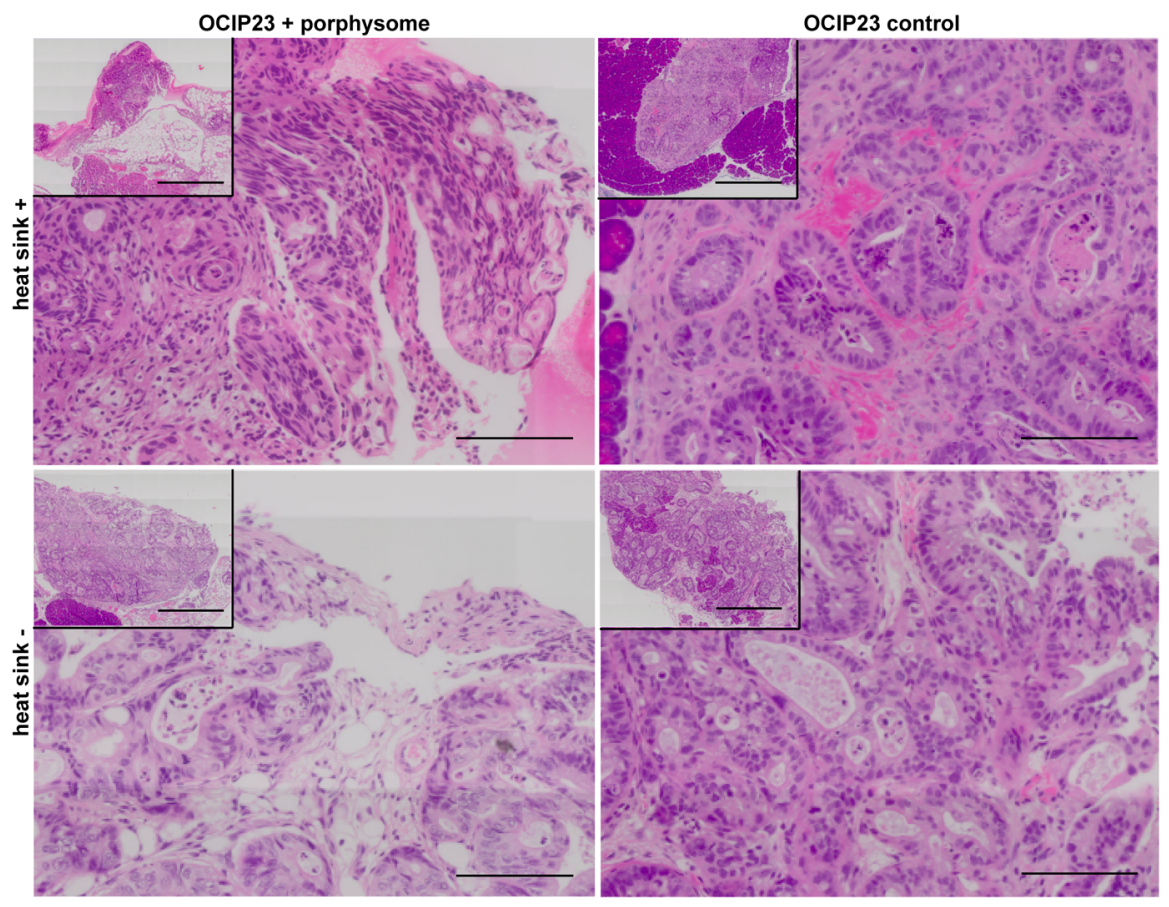

Fig. 6 Representative images from H\&E stained OCIP23 tumor tissue slices following laser irradiation (scale bar $100 \mu \mathrm{m}$ ). Heat sink+ samples indicate light irradiation with open abdomen (light dose: $468 \mathrm{~J} / \mathrm{cm}^{2}$ over $220 \mathrm{~s}$ to reach $70^{\circ} \mathrm{C}$ target temperature). Heat sink- samples indicate that tumors were raised from their natural seat in the open abdomen to reduce heat diffusion through tissues and vasculature during light irradiation (light dose: $137 \mathrm{~J} / \mathrm{cm}^{2}$ over $70 \mathrm{~s}$ to reach $70^{\circ} \mathrm{C}$ target temperature). Inset: low-magnification view, scale bar $500 \mu \mathrm{m}$.

Though immediate direct thermal damage may not be achieved, exposure to hyperthermic conditions can sensitize cells to adjunct chemotherapy and could contribute to overall treatment efficacy. ${ }^{35,36}$ Furthermore, thorough characterization of the spatial distribution of heat diffusion in this cancer model is significant due to proximity of tumors to vital organs to which nonspecific damage could result in treatment-associated morbidity in the clinical situation.

In summary, porphysomes have been demonstrated as effective high-payload porphyrin delivery vehicles by reproducibly accumulating in xenograft tumors of a highly clinically relevant pancreatic cancer model, as revealed by fluorescence imaging and biodistribution studies. The presence of porphyrin moieties in tumors appears to decrease the requisite light dose for attaining the therapeutic temperature threshold during PTT. Furthermore, histological analyses of laser treated tumor tissue microstructure corroborate the specificity of the porphysomeenhanced PTT.

In the administration of thermal ablative therapies for reduction of tumor burden in well-perfused abdominal organs such as the liver or kidneys, it has been reported that the total fluence and energy deposition rate required to reach therapeutic temperatures is complicated by the fact that perfusion may remove heat from the tumor region more rapidly than it can be generated. ${ }^{5,8,9}$ Conversely, this heat-sink effect may be advantageous for reducing treatment-associated morbidity and mortality by contributing to retention of surrounding vessel patency and preservation of other surrounding structures. It is notable that the preliminary study of the heat-sink effect herein shows a measureable change in the rate of tissue heating while light and sensitizer dosimetry are held constant in a tumor model that recapitulates the clinical features of pancreatic cancer. Most notably, incorporating biocompatible photothermal sensitizers such as porphysomes into PTT protocols also brings promise by decreasing the required light dose, and promoting spatial confinement of coagulative necrosis to the target tumor tissue, though larger scale studies are required to unambiguously identify the specificity of these effects. While pancreatic cancer is frequently a metastatic disease, the development of novel strategies for reduction of primary tumor burden brings significant potential to improve patient quality-of-life as well as survival.

\section{Acknowledgments}

The authors acknowledge Israel Veilleux for contribution to laser irradiation optics and Warren Foltz for MR images. This work was supported by the Canadian Institute of Health Research, the Patrick Hodgson Family Research Fund at Princess Margaret Cancer Centre and the Knudson Postdoctoral Fellowship Program.

\section{References}

1. C. A. Iacobuzio-Donahue et al., "DPC4 gene status of the primary carcinoma correlates with patterns of failure in patients with pancreatic cancer," J. Clin. Oncol. 27(11), 1806-1813 (2009).

2. D. P. Ryan, T. S. Hong, and N. Bardeesy, "Pancreatic adenocarcinoma," N. Engl. J. Med. 371(11), 1039-1049 (2014).

3. M. Youl et al., "Induction gemcitabine plus concurrent gemcitabine and radiotherapy for locally advanced unresectable or resected pancreatic cancer," Clin. Oncol. 26(4), 203-209 (2014).

4. S. J. E. Rombouts et al., "Systematic review of innovative ablative therapies for the treatment of locally advanced pancreatic cancer," $\mathrm{Br} . J$. Surg. 102(3), 182-193 (2015). 
5. K. F. Chu and D. E. Dupuy, "Thermal ablation of tumours: biological mechanisms and advances in therapy," Nat. Rev. Cancer 14(3), 199-208 (2014).

6. G. Jori and J. D. Spikes, "Photothermal sensitizers: possible use in tumor therapy," J. Photochem. Photobiol. B Biol. 6(1-2), 93-101 (1990).

7. J. Fang and Y.-C. Chen, "Nanomaterials for photohyperthermia: a review," Curr. Pharm. Des. 19(37), 6622-6634 (2013).

8. C. Brace, "Thermal tumor ablation in clinical use," Inst. Electr. Electron. Eng. Pulse 2(5), 28-38 (2011).

9. L. Thanos et al., "Overcoming the heat-sink phenomenon: successful radiofrequency thermal ablation of liver tumors in contact with blood vessels," Diagn. Interventional Radiol. 14(1), 51-56 (2008).

10. H.-W. Huang, "Influence of blood vessel on the thermal lesion formation during radiofrequency ablation for liver tumors," Med. Phys. 40(7), 073303 (2013).

11. N. Bhardwaj et al., "A comparative histological evaluation of the ablations produced by microwave, cryotherapy and radiofrequency in the liver," Pathology 41(2), 168-172 (2009).

12. B. B. Frericks et al., "Influence of intrahepatic vessels on volume and shape of percutaneous thermal ablation zones: in vivo evaluation in a porcine volume," Invest. Radiol. 43(4), 211-218 (2008).

13. J. F. Lovell et al., "Porphysome nanovesicles generated by porphyrin bilayers for use as multimodal biophotonic contrast agents," Nat. Mater. 10(4), 324-332 (2011).

14. M. T. Huggett et al., "Phase I/II study of verteporfin photodynamic therapy in locally advanced pancreatic cancer," Br. J. Cancer 110(7), 1698-1704 (2014).

15. Q. Chang et al., "Hypoxia predicts aggressive growth and spontaneous metastasis formation from orthotopically grown primary xenografts of human pancreatic cancer," Cancer Res. 71(8), 3110-3120 (2011).

16. I. Lohse et al., "Assessment of hypoxia in the stroma of patientderived pancreatic tumor xenografts," Cancers (Basel) 6(1), 459-471 (2014).

17. Y. Yang, "Upconversion nanophosphors for use in bioimaging, therapy, drug delivery and bioassays," Microchim. Acta 181(3-4), 263-294 (2014).

18. Z. Liu et al., "Carbon materials for drug delivery and cancer therapy," Mater. Today 14(7-8), 316-323 (2011).

19. Y. Zhang, C. Y. Ang, and Y. Zhao, "Polymeric nanocarriers incorporating near-infrared absorbing agents for potent photothermal therapy of cancer," Polym. J. 48, 589-603 (2016).

20. X. Huang and M. A. El-Sayed, "Gold nanoparticles: optical properties and implementations in cancer diagnosis and photothermal therapy," J. Adv. Res. 1(1), 13-28 (2010).

21. K. A. Willets and R. P. Van Duyne, "Localized surface plasmon resonance spectroscopy and sensing," Ann. Rev. Phys. Chem. 58, 267-297 (2007).
22. S. Link and M. A. El-Sayed, "Shape and size dependence of radiative, non-radiative and photothermal properties of gold nanocrystals," Int. Rev. Phys. Chem. 19(3), 409-453 (2000).

23. H. S. Choi et al., "Renal clearance of nanoparticles," Nat. Biotechnol. 25(10), 1165-1170 (2007).

24. P. K. Jain et al., "Calculated absorption and scattering properties of gold nanoparticles of different size, shape, and composition: applications in biological imaging and biomedicine," J. Phys. Chem. B 110(14), 7238-7248 (2006).

25. Q. Chang et al., "Tumor-stroma interaction in orthotopic primary pancreatic cancer xenografts during hedgehog pathway inhibition," Int. J. Cancer 133(1), 225-234 (2013).

26. D. Xie and K. Xie, "Pancreatic cancer stromal biology and therapy," Genes Dis. 2(2), 133-143 (2015).

27. G. Allegretti et al., "Magnetic resonance-based thermometry during laser ablation on ex-vivo swine pancreas and liver," Med. Eng. Phys. 37(7), 631-641 (2015).

28. L. Mocan et al., "Selective ex-vivo photothermal ablation of human pancreatic cancer with albumin functionalized multiwalled carbon nanotubes," Int. J. Nanomed. 6, 915-928 (2011).

29. T. Patino et al., "Multifunctional gold nanorods for selective plasmonic photothermal therapy in pancreatic cancer cells using ultra-short pulse near-infrared laser irradiation," Nanoscale 7(12), 5328-5337 (2015).

30. Y. Guo et al., "Photothermal ablation of pancreatic cancer cells with hybrid iron-oxide core gold-shell nanoparticles," Int. J. Nanomed. 8, 3437-3446 (2013).

31. W. Chen et al., "Targeting pancreatic cancer with magneto-fluorescent theranostic gold nanoshells," Nanomedicine 9(8), 1209-1222 (2014).

32. G. Tian et al., "Multifunctional Rb-x WO3 nanorods for simultaneous combined chemo-photothermal therapy and photoacoustic/CT imaging," Small 10(20), 4160-4170 (2014).

33. C. S. Jin et al., "Ablation of hypoxic tumors with dose-equivalent photothermal, but not photodynamic, therapy using a nanostructured porphyrin assembly," ACS Nano 7(3), 2541-2550 (2013).

34. T. W. Liu et al., "Inherently multimodal nanoparticle-driven tracking and real-time delineation of orthotopic prostate tumors and micrometastases," ACS Nano 7(5), 4221-4232 (2013).

35. R. D. Issels, "Hyperthermia adds to chemotherapy," Eur. J. Cancer 44(17), 2546-2554 (2008).

36. P. Wust et al., "Hyperthermia in combined treatment of cancer," Lancet Oncol. 3(8), 487-497 (2002).

37. S. E. Mills, Ed., Histology for Pathologists, 4th ed., pp. 689-690, Lippincott Williams \& Wilkins, Philadelphia, Pennsylvania (2012).

38. H. Schoellnast et al., "Interstitial laser-induced thermotherapy of the lung: evaluation of the influence of ablation continuity on ablation size in a swine model," Cancer Imaging 13(1), 8-13 (2013).

Biographies for the authors are not available. 